\title{
Edge and Surface States in the Quantum Hall Effect in Graphene
}

\author{
A. H. Castro Neto ${ }^{1}$, F. Guinea ${ }^{1,3}$, and N. M. R. Peres ${ }^{1,2}$ \\ ${ }^{1}$ Department of Physics, Boston University, 590 Commonwealth Avenue, Boston, MA 02215, USA \\ ${ }^{2}$ Center of Physics and Department of Physics, Universidade do Minho, P-4710-057, Braga, Portugal and \\ ${ }^{3}$ Instituto de Ciencia de Materiales de Madrid, CSIC, Cantoblanco E28049 Madrid, Spain
}

\begin{abstract}
We study the integer and fractional quantum Hall effect on a honeycomb lattice at half-filling (graphene) in the presence of disorder and electron-electron interactions. We show that the interactions between the delocalized chiral edge states (generated by the magnetic field) and Andersonlocalized surface states (created by the presence of zig-zag edges) lead to edge reconstruction. As a consequence, the point contact tunneling on a graphene edge has a non-universal tunneling exponent, and the Hall conductivity is not perfectly quantized in units of $e^{2} / h$. We argue that the magnetotransport properties of graphene depend strongly on the strength of electron-electron interactions, the amount of disorder, and the details of the edges.
\end{abstract}

PACS numbers: 73.43.-f; 71.55.-i; 71.10.-w

\section{INTRODUCTION}

Recent progress in development of gate and magnetic field controlled, two-dimensional (2D), graphitic devices ${ }^{1.2}$ has not only opened doors for Carbon microelectronics, but also renewed the interest in the study of strongly interacting, low dimensional, electronic systems. Graphene is a 2D Carbon material with a honeycomb lattice and one electron per $\pi$ orbital (half-filled band), whose elementary excitations are Dirac electrons that reside at the corners of the Brillouin zone. These excitations have linear dispersion relation, $\epsilon_{ \pm}(\mathbf{k})= \pm v_{\mathrm{F}}|\mathbf{k}|$, with a characteristic Dirac-Fermi velocity $v_{\mathrm{F}}$. All electronic properties of graphene are determined by the physics of Dirac fermions which are quite anomalous when compared to the ones found in ordinary electrons: the absence of dynamical screening ${ }^{3}$, a non-Fermi liquid quasi-particle lifetime ${ }^{4}$, and anomalous scattering by impurities ${ }^{\underline{5}}$. Moreover, in the presence of strong disorder, graphite samples (which are obtained from stacking of graphene layers) become ferromagnetic $\frac{6.7}{}$ indicating the important interplay between disorder and electronelectron interactions in these materials. We have recently shown that because of the low dimensionality, disorder, particle-hole asymmetry, and strong Coulomb interactions, graphene presents the phenomenon of self-doping in which extended defects, such as dislocations, disclinations, edges, and micro-cracks, shift the chemical potential away from the Dirac point to produce electron or hole pockets ${ }^{8}$. The presence of localized disorder, such as vacancies and adatoms, leads also to non-trivial physical effects that must be understood in order to interpret the data correctly.

In this letter we investigate transport properties of graphene under high magnetic fields in the Hall geometry shown in Fig. 1. When a high magnetic field, $B$, is applied to a 2D material, the electronic bulk develops Landau levels which in the case of Dirac fermions have energy $\epsilon_{n}= \pm v_{\mathrm{F}} l_{\mathrm{B}}^{-1} \sqrt{n}$, where $n$ is a positive integer, $l_{\mathrm{B}}=\sqrt{\Phi_{0} / B}$ is the cyclotron radius, and $\Phi_{0}=c h / e$

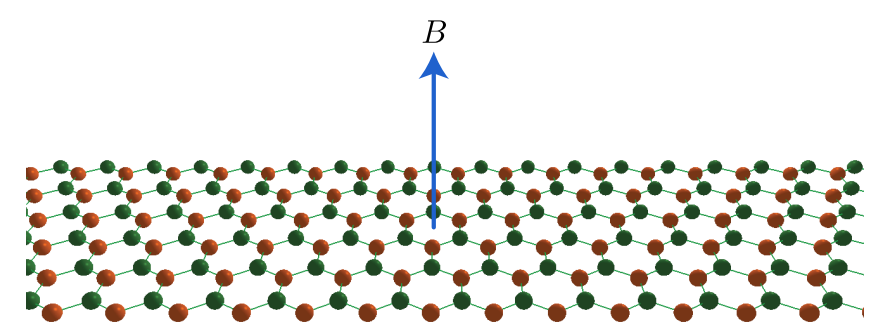

FIG. 1: (color online) Perspective view of the Hall geometry used in our calculations with periodic boundary conditions in one direction and zig-zag edges in the other. The magnetic field $B$ is applied perpendicular to the graphene plane.

is flux quanta. Thus, the bulk of the system is gapped by the cyclotron energy scale, $\hbar \omega_{c}=\sqrt{2} v_{\mathrm{F}} \hbar / l_{\mathrm{B}}$ (which is much larger than the Zeeman energy, $g \mu_{B} B$, where $g \approx 2$ and $\mu_{B}$ the Bohr magneton ${ }^{9}$ ).

In the integer quantum Hall effect (IQHE), the bulk states are gapped and localized due to the disorder, and the electronic conduction in a Hall bar occurs through its edges ${ }^{10}$. In the case of graphene, due to the Dirac fermion nature of its carriers, the Hall conductivity is given by 8.11 :

$$
\sigma_{\mathrm{IQHE}}=(2 N+1) \frac{2 e^{2}}{h},
$$

where $N$ is an integer, and $e$ is the electron charge. Besides supporting bulk states a graphene Hall bar, such as the one shown in Fig. 10 also supports surface states ${ }^{12.13}$. The result (11) is only valid if the surface states do not contribute to the conduction.

In this paper we study the integer and fractional quantum Hall effect (FQHE) in graphene taking into account 
the edge and surface states of a graphene Hall bar. We show that even in the presence of disorder, when the surface states become localized, they have a direct effect in the magneto-transport. We show that the quantization of the conductivity, as given in (1), is not exact by the presence of surface states and becomes dependent on the details of the sample such as the amount of disorder. Our prediction for the FQHE can be verified experimentally in graphitic devices 1.2 .

The paper is organized as follows: in Section III we present the Hamiltonian for the problem and show how surface and edge Hall modes originate in a graphene Hall bar; in Section III we discuss the theory for the edge modes; Section [V] contains the theory for the surface states in the presence of disorder and electron-electron interactions; in Section $\mathrm{V}$ we discuss the Coulomb interaction between edge and surface states and the phase diagram of a graphene Hall edge in the presence of disorder and electron-electron interactions; Section VI contains our conclusions. We have also included one appendix with the details of the calculations.

\section{THE MODEL}

The kinetic energy of electrons in graphene is described by the Hamiltonian (from now on, we use units such that $\left.\hbar=1=k_{B}\right)$ :

$$
\mathcal{H}_{\text {kinetic }}=-t \sum_{\sigma ;\langle i, j\rangle} c_{i, \sigma}^{\dagger} c_{j, \sigma}+t^{\prime} \sum_{\sigma ;\langle\langle i, j\rangle\rangle} c_{i, \sigma}^{\dagger} c_{j, \sigma}+\text { h.c. }
$$

where $c_{i, \sigma}\left(c_{i, \sigma}^{\dagger}\right)$ annihilates (creates) electrons at the site $\mathbf{R}_{i}$ with spin $\sigma(\sigma=\uparrow, \downarrow), t$ and $t^{\prime}$ are the nearest neighbor and next-nearest neighbor hopping energies, respectively. At long-wavelengths the electronic dispersion is given by:

$$
\epsilon_{ \pm}(\mathbf{k}) \approx 3 t^{\prime} \pm v_{\mathrm{F}}|\mathbf{k}|+\frac{9 t^{\prime} a^{2}|\mathbf{k}|^{2}}{4}
$$

where

$$
v_{\mathrm{F}}=\frac{3 t a}{2}
$$

In the presence of a magnetic field $\mathbf{B}$ the electronic hopping between sites $\mathbf{R}_{i}$ and $\mathbf{R}_{j}$ is modified via the Peierls substitution, that is, we rewrite the hopping as $t_{i j} \exp \left\{i \varphi_{i j}\right\}$ with $\varphi_{i j}=2 \pi \int_{\mathbf{R}_{i}}^{\mathbf{R}_{j}} \mathbf{A}(\mathbf{r}) \cdot d \mathbf{r} / \Phi_{0}$, where $\mathbf{A}(\mathbf{r})$ is the vector potential $(\mathbf{A}=\nabla \times \mathbf{B})$.

The spectra of the problem for a graphene Hall bar in the presence of a magnetic field can be calculated exactly by solving an eigenvalue equation 14 . In Fig. 2 we show the spectra and wavefunctions for a graphene Hall bar that is periodic along the direction parallel to the edges and 300 lattice spacing wide, such as the one shown in Fig. 1 described in the tight-binding limit with $t^{\prime}=0.2 t$ in the presence of a magnetic field such that the magnetic flux per hexagon, $\Phi$, is $\Phi=10^{-3} \Phi_{0}$.
One can clearly see two types of states: bulk states that become edge states because of the finite size of the Hall bar and a surface state localized at the edge of the sample. In the presence of $t^{\prime}$ (that breaks particle-hole symmetry) the surface mode is dispersive with a characteristic velocity:

$$
v_{\mathrm{S}} \sim t^{\prime} a
$$

while edge states have a velocity:

$$
v_{\mathrm{E}} \sim t a
$$

where $a \approx \sqrt{3} \times 1.42 \AA$ is the lattice spacing.

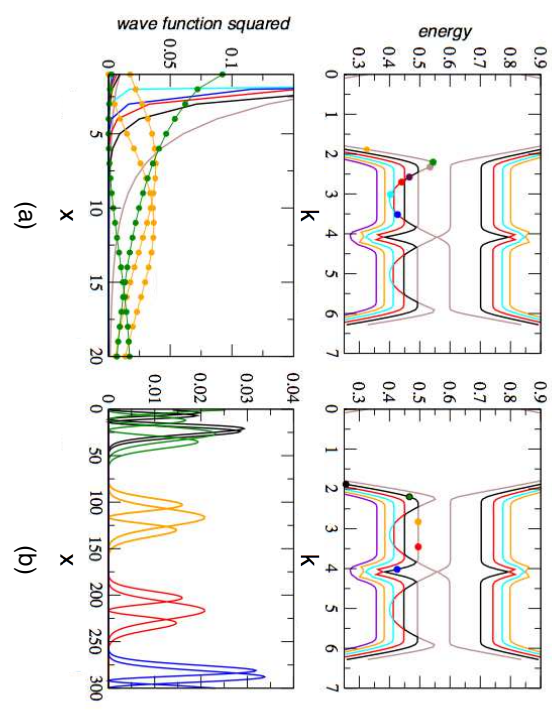

FIG. 2: (color online) Electronic spectra (top) and wavefunctions (bottom) of a graphene Hall bar as function of the momentum $k$ parallel to the edge. For each bullet in the top panels (going from left to right), we plot in the bottom panel the wavefunction squared as function of the distance $x$ to the edge. Note that the two top panels represent the same set of bands. (a) and (b): Surface states. (c) and (d): bulk Landau levels. Energy given in units of $t$ and distance in units of the lattice spacing $a$.

\section{EDGE STATES}

In order to study the edge states it is conceptually simple to consider a large graphene droplet instead of a Hall bar ${ }^{15}$. When a large magnetic field $\mathbf{B}=B \mathbf{z}$, is applied perpendicular to the graphene, a persistent current $\mathbf{J}$ flows along the edge of the droplet, being confined by an electric field $\mathbf{E}$ created by the termination of the graphene droplet. The value of the current is given by:

$$
\mathbf{J}=\sigma_{x y} \mathbf{z} \times \mathbf{E}
$$

where

$$
\sigma_{x y}=\nu \frac{2 e^{2}}{h}
$$


is the Hall conductivity. Here, $\nu=2 \pi \ell_{B}^{2} \delta$, is the filling fraction of the droplet, $\delta$ is the $2 \mathrm{D}$ electronic density (away from half-filling). Notice, therefore, that the electrons will drift along the edge with velocity $v=E c / B$ and, hence, the $2 \mathrm{D}$ electronic density along the edge, $\rho_{e}(x, t)=\delta n(x)$ (where $n(x)$ is the displacement of the edge) obeys the equation:

$$
\partial_{t} \rho_{e}(x, t)-v \partial_{x} \rho_{e}(x, t)=0,
$$

which describes a chiral motion (classically, $\rho_{e}(x, t)=$ $\left.\rho_{e}(x-v t)\right)$.

The classical problem can be quantized in terms of the Fourier components of the density:

$$
\rho_{k}=\frac{1}{\sqrt{L}} \int d x e^{i k x} \rho_{e}(x),
$$

where $L$ is the circumference of the edge, by canonical commutation:

$$
\left[\rho_{k}, \rho_{-k^{\prime}}\right]=\frac{\nu}{2 \pi} k \delta_{k, k^{\prime}} .
$$

The Hamiltonian of the edge waves is then simply:

$$
H=\frac{2 \pi v}{\nu} \sum_{k>0} \rho_{k} \rho_{-k} .
$$

The edge fermion operator can be constructed from the density operators via a bosonic field, $\phi(x)$, such that:

$$
\rho_{e}(x)=\frac{1}{2 \pi} \partial_{x} \phi(x) .
$$

The electron operator, $\Psi_{e}(x)$, is given by the Mandelstam construction:

$$
\Psi_{e}(x) \propto e^{\frac{i}{\nu} \phi(x)},
$$

that preserves the commutation relations between the electron and the density operators:

$$
\left[\rho_{e}(x), \Psi_{e}^{\dagger}(y)\right]=\delta(x-y) \Psi_{e}^{\dagger}(x) .
$$

This result indicates that the operator carries charge $e$, as required. Furthermore, it is easy to show that:

$$
\Psi_{e}(x) \Psi_{e}(y)=(-1)^{1 / \nu} \Psi_{e}(y) \Psi_{e}(x),
$$

and, thus, by the Pauli principle we must require that:

$$
\nu=\frac{1}{m},
$$

where $m$ is an odd integer. The constraint (17), when applied to (8), gives the quantization of Hall conductivity. Thus, it is clear that this construction can only describe Laughlin's main sequence. For more complicated QHE sequences one has to use multiple edge states ${ }^{8}$. In this work we focus on the case given in (17).

It is also easy to show that:

$$
\langle\phi(x) \phi(0)\rangle=-\nu \ln (x)+\text { constant },
$$

and, hence,

$$
\left\langle\Psi_{e}^{\dagger}(x) \Psi_{e}(0)\right\rangle \propto e^{\frac{1}{\nu^{2}}\langle\phi(x) \phi(0)\rangle} \propto \frac{1}{x^{m}},
$$

as expected. In terms of path integrals, the action for the 1D chiral Luttinger liquid action reads:

$$
S_{\text {edge }}=\int_{x, t} \frac{m}{4 \pi}\left[\partial_{t} \phi \partial_{x} \phi-v_{\mathrm{E}}\left(\partial_{x} \phi(x)\right)^{2}\right],
$$

where $\phi(x, t)$ is a bosonic chiral field along the edge at position $x$ and time $t$.

This construction stresses the robustness of the Hall effect: the edge state being chiral in nature, cannot suffer any backscattering. It is exactly the electron-electron backscattering interaction that creates density wave and superconducting states, and the impurity backscattering interaction that leads to Anderson localization ${ }^{16}$. Therefore, chiral edge states iarenot influenced either by electron-electron interactions or disorder. Finally we note that the exponent $m$ is determined entirely by the bulk of the system and has topological origin 15 .

In the next section we are going to show that forward scattering interactions between chiral edge states and surface states modify the chiral action (20) introducing instabilities in the chiral states at finite momenta. If this is the case, it is clear that the relation (17) (or (19) will not hold, spoiling the perfect quantization of the Hall conductivity.

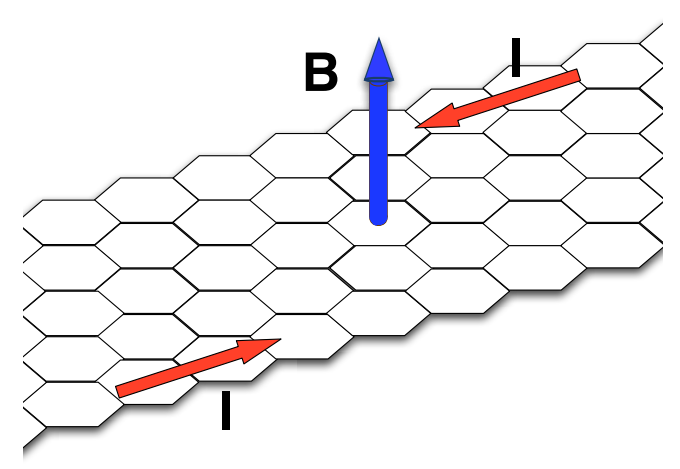

FIG. 3: (color online) Perspective view of edge currents, $I$, on a graphene Hall bar. 


\section{SURFACE STATES AND DISORDER}

The graphene surface state is also a $1 \mathrm{D}$ state that can be described by a non-chiral Luttinger liquid action ${ }^{16}$ :

$$
S_{\text {surf }}=\int_{x, t} \frac{1}{2 \pi K}\left[\frac{1}{u}\left(\partial_{t} \theta(x)\right)^{2}-u\left(\partial_{x} \theta(x)\right)^{2}\right],
$$

where $\theta(x, t)$ is a bosonic field at the edge,

$$
u=v_{\mathrm{S}}\left[1-f^{2} /\left(4 \pi^{2} v_{\mathrm{S}}^{2}\right)\right]^{1 / 2},
$$

is the renormalized Luttinger liquid velocity ( $f$ is the electron-electron forward scattering coupling constant), and

$$
K=\left[\left(1-f /\left(2 \pi v_{\mathrm{S}}\right)\right) /\left(1+f /\left(2 \pi v_{\mathrm{S}}\right)\right)\right]^{1 / 2},
$$

is the Luttinger parameter that measures the decay of the surface correlation functions ${ }^{16}$. The surface density, $\rho_{s}(x)$, is written as:

$$
\rho_{s}(x)=\frac{1}{2 \pi} \partial_{x} \theta(x) .
$$

Notice that unlike the edge mode, the surface mode is sensitive to the electron-electron interactions and also disorder.

While the problem without disorder can be easily studied theoretically ${ }^{17}$ (see below), in most of this paper we focus on the realistic case of a disordered edge, such as the one shown in Fig. 4 Missing Carbon atoms at the edge of a graphene hall bar effectively "cuts" the electronic wavefunction and leads to lateral confinement of the electrons. The lateral confinement of the electrons leads to the discretization of the surface state energy levels, as one would have for a particle moving in a 1D box. Hence, these electronic puddles have a characteristic excitation energy scale, or gap, of the order of

$$
\Delta(\ell) \sim \frac{v_{S}}{\ell} \sim \frac{t^{\prime} a}{\ell},
$$

where $\ell$ is the size of the $1 \mathrm{D}$ domain. This effect is clearly seen in scanning tunneling microscopy (STM) studies of the surface of graphite 18 .

The presence of disorder at the edge of the graphene Hall bar leads to a back-scattering of the surface electron states which can be written as:

$$
S_{\mathrm{BS}}=\int_{x, t}\left(V(x) e^{i\left(\sqrt{2} \theta(x, t)+2 k_{F} x\right)}+\text { h.c. }\right),
$$

where $V(x)$ is the scattering potential (notice that only the $2 k_{F}$ component of the disorder potential contributes at low energies, and that the forward scattering part of the potential is irrelevant). In the presence of disorder $V(x)$ is a random variable with probability: $\mathcal{P}[V(x)] \propto$ $\exp \left\{-\int d x|V(x)|^{2} / V_{0}^{2}\right\}$ so that, after averaging over disorder: $\left[V(x) V^{*}(y)\right]_{\text {disorder }}=V_{0}^{2} \delta(x-y)$. Hence, $V_{0}$ provides a measure of the amount of disorder in the system.
One possible way of dealing with (26) is via a replicatrick $^{19}$. In this case, one has to add a new term to the Luttinger liquid action (21):

$$
S_{\mathrm{BS}}=-V_{0}^{2} \int_{x, t, t^{\prime}} \sum_{i, j} \cos \left[\sqrt{2}\left(\theta_{i}(x, t)-\theta_{j}\left(x, t^{\prime}\right)\right)\right]
$$

where $\theta_{i}(x, t)$ indicates the field $\theta$ in the $i^{\text {th }}$ replica. Notice that this term is highly non-local because of the quenched disorder. In the absence of edge modes, the full action (21) plus (27) can be understood via a renormalization group (RG) calculation assuming the disorder to be weak, that is, we define a dimensionless disorder strength, $D$ :

$$
D \approx \frac{2 V_{0}^{2} a^{2}}{\pi u^{2}}
$$

and obtain the RG equations ${ }^{19}$ in leading order in $\delta$ :

$$
\begin{aligned}
\partial_{\ell} K^{-1} & =\frac{D}{2}, \\
\partial_{\ell} D & =(3-2 K) D,
\end{aligned}
$$

where $\ell=\ln \left(W_{0} / W\right)$ is the $\mathrm{RG}$ scale ( $W$ is the running energy cut-off of the bosons, $W_{0} \sim t^{\prime}$ is the bare cut-off). It is easy to see that disorder is irrelevant if $K>3 / 2$, and it is relevant if $K<3 / 2$, under the RG flow. Notice that from (23) we have $K<1$ for repulsive interactions $(f>$ 0 ) and therefore the above RG indicates that disorder always flows to strong coupling, $D(\ell \rightarrow \infty) \rightarrow \infty$, and strong interactions, $K(\ell \rightarrow \infty) \rightarrow 0$, as expected. This result implies that the $\mathrm{RG}$ breaks down at certain scale where $D\left(\ell^{*}\right) \approx 1$ and the surface states become Anderson localized. The localization scale can be estimated from (29) by introducing a localization length, $\xi$, so that: $\ell^{*}=$ $\ln \left(W_{0} /\left(u \xi^{-1}\right)\right.$, and from (29) one finds:

$$
\xi \approx a D_{0}^{-1 /\left(3-2 K^{*}\right)}
$$

where $D_{0}$ is the bare amount of disorder in the system and $K^{*} \approx K\left(\ell^{*}\right)$. Notice that this result indicates that there is a characteristic energy scale, $E_{\mathrm{loc}}$, associated with the disorder which is of the order of $E_{\mathrm{loc}}(\xi) \sim v_{S} / \xi \sim t^{\prime} D_{0}^{1 /\left(3-2 K^{*}\right)}$. Direct comparison with (25) shows that $\xi$ can be thought as the typical size of the electronic puddles at the edge with a gap in the energy spectrum given by $E_{\text {loc }}$.

This result indicates that the bosonic correlations at larger distances decay exponentially with $\xi$ (the time correlations are also short ranged with a characteristic time scale $\left.\tau_{\text {loc }} \sim 1 / E_{\text {loc }} \sim \xi / v_{\mathrm{S}}\right)$. In this case, it is reasonable to replace the Luttinger liquid action (21) by:

$$
S_{\mathrm{s}} \approx-\int_{x, t} \frac{u}{2 \pi K}\left[\frac{1}{\xi^{2}} \theta^{2}(x)+\left(\partial_{x} \theta(x)\right)^{2}\right],
$$

so that: $[\langle\theta(x, t) \theta(0,0)\rangle]_{\text {disorder }} \approx \delta(t) e^{-x / \xi}$, for $x \gg \xi$ and $t \gg \tau_{\text {loc }}$. 


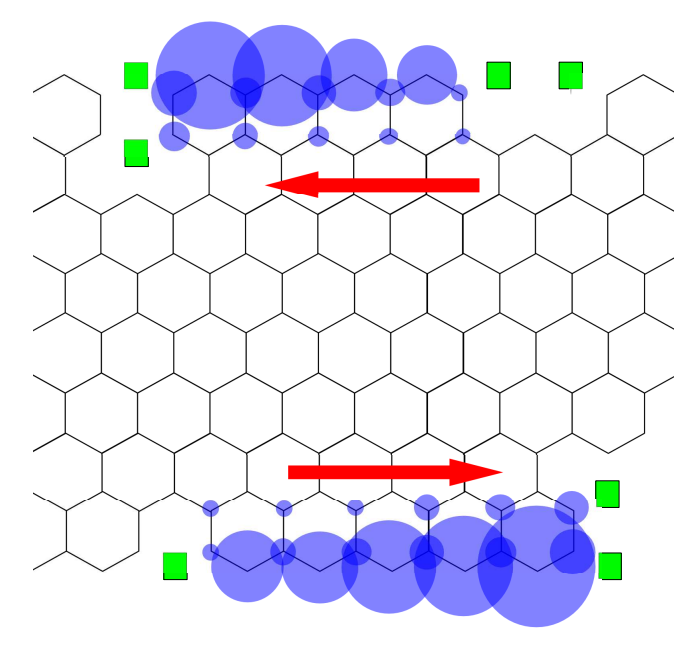

FIG. 4: (color online) Schematic representation of a graphene Hall bar with a disordered zig-zag edge and its electronic puddles (see ref. [18]). Squares represent missing Carbon atoms, the circles' radii represent the amplitude of the localized surface electronic wavefunction and the formation of electronic puddles. The arrows show the direction of the edge currents.

\section{ELECTRON-ELECTRON INTERACTIONS AND EDGE RECONSTRUCTION}

Because the edge and the surface states are confined to a small region in space they interact with each other via a Coulomb interaction. This interaction leads to a new term in the problem:

$$
S_{\text {coupling }}=-\frac{\lambda}{\pi} \int_{x, t} \partial_{x} \theta \partial_{x} \phi
$$

where,

$$
\lambda=\frac{e^{2} a}{4 \pi \ell_{\mathrm{B}}}
$$

is the strength of the electron-electron coupling.

\section{A. Clean surface states}

Let us consider first the case of a clean surface state interacting with a chiral edge state. The edge state is described by the chiral Luttinger liquid Hamiltonian given by:

$$
H_{\chi}=\sum_{q>0} q v_{S} a_{q}^{\dagger} a_{q}
$$

where $a_{q}\left(a_{q}^{\dagger}\right)$ annihilates (creates) a chiral boson with momentum $q$, while the surface state is described by the
Hamiltonian:

$H_{L L}=\sum_{q>0} q v_{E}\left(b_{q}^{\dagger} b_{q}+b_{-q}^{\dagger} b_{-q}\right)+\sum_{q>0} \bar{V}(q)\left(b_{q}^{\dagger} b_{q}+b_{-q}^{\dagger} b_{-q}\right)$

where $\bar{V}(q)$ is the surface state forward scattering interaction. Let us consider a generic surface-edge interaction potential, $V(q)$, and the interaction Hamiltonian:

$$
H_{\chi, L L}=\sum_{q>0} V(q)\left[a_{q}^{\dagger}\left(b_{q}^{\dagger}+b_{-q}\right)+a_{q}\left(b_{-q}^{\dagger}+b_{q}\right)\right] .
$$

The Hamiltonian $H_{L L}$ can by diagonalized via a Bogoliubov-Valatin transformation ${ }^{20}$, leading to new bosonic modes $c_{q}$ and $d_{q}$ :

$$
H_{L L}=\sum_{q>0} E(q)\left(c_{q}^{\dagger} c_{q}+d_{q}^{\dagger} d_{q}\right)
$$

with $E(q)=\sqrt{q^{2} v_{E}^{2}-[\bar{V}(q)]^{2}}$. Under the transformation the interaction Hamiltonian becomes:

$$
H_{\chi, L L}=\sum_{q>0} \tilde{V}(q)\left[a_{q}^{\dagger}\left(d_{q}^{\dagger}+c_{q}\right)+a_{q}\left(d_{q}+c_{q}^{\dagger}\right)\right]
$$

with $\tilde{V}(q)=V(q)\left[\cosh \left(\lambda_{q}\right)-\sinh \left(\lambda_{q}\right)\right], \cosh \left(2 \lambda_{q}\right)=$ $q v_{F}(q) / E(q)$, and $\sinh \left(2 \lambda_{q}\right)=\bar{V}(q) / E(q)$. The Hamiltonian composed by the sum of (34), (37), and (38), is the form of the effective Hamiltonian described in ref. [21], and can be diagonalized by a generalized BogoliubovValatin transformation ${ }^{21.23}$. Introducing a spinor field $\Psi^{\dagger}=\left(a_{q}^{\dagger}, c_{q}^{\dagger}, d_{q}\right)$ the total Hamiltonian reads:

$$
H=\sum_{q>0} \Psi^{\dagger} D \Psi-\sum_{q>0} E(q)
$$

where $D$ in the grand-dynamical matrix ${ }^{22}$. The Hamiltonian (39) has the form (apart from constant terms):

$$
H=\sum_{q>0}\left[\omega_{\alpha}(q) \alpha_{q}^{\dagger} \alpha_{q}+\omega_{\beta}(q) \beta_{q}^{\dagger} \beta_{q}+\omega_{\gamma}(q) \gamma_{q}^{\dagger} \gamma_{q}\right]
$$

where, after diagonalization, and the new quasiparticles operators read:

$$
\Psi^{\dagger}\left(T^{\dagger}\right)^{-1}=\left(\alpha_{q}^{\dagger}, \beta_{q}^{\dagger}, \gamma_{q}\right)
$$

where the matrix $T$ as the form considered in ref. 21 23].

The diagonalization of the Hamiltonian (39) amounts to find the values of the angles $\theta, \phi$, and $\eta$ such that the matrix $T^{\dagger} A T$ has non-zero diagonal elements only. All matrix elements of matrix $A$ are given in appendix A As in ref. 21], the relation $\omega_{\alpha}(q)+\omega_{\beta}(q)-\omega_{\gamma}(q)=q v_{S}$ holds. We have solved the eigenvalue problem for two different kinds of electron-electron potentials: $(i)$ a contact potential given by $V(x)=V_{0} a \delta(x)$; $(i i) V(x)=$ $V_{0} \exp (-(|x| / a))$. The dispersion of the bosonic modes is shown in Fig. 5] Although both potentials are short 


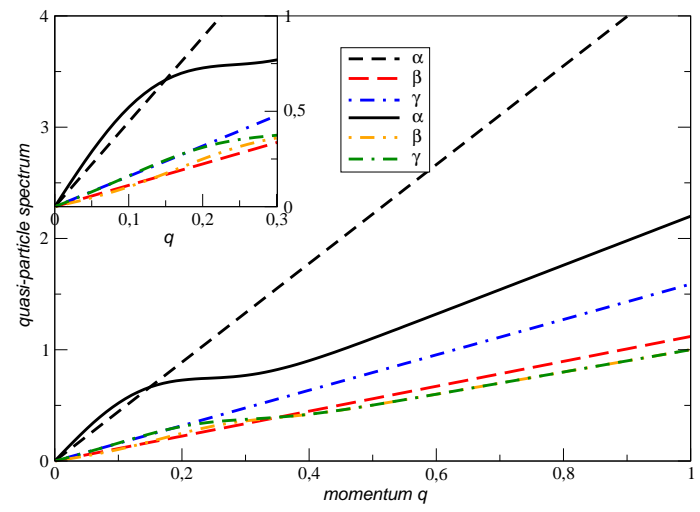

FIG. 5: (color online) Quasi-particles spectrum for the two potentials considered in the text: $(i)$ dashed line; $(i i)$ solid line. The parameters are $V_{1}=1.1, V_{2}=1.2, V_{3}=1.3$, $a_{1}=10.1, a_{2}=10.2, a_{3}=10.3, v_{S}=2.2, v_{E}=1$.

ranged, case (ii) introduces a momentum scale $k_{s} \sim$ $1 / a$ where the spectrum deviates significantly from the sound-like behavior obtained with potential $(i)$. It is clear from these results that although the coupling between the edge modes and surface modes alters the dispersion at finite wavelengths it does not lead to any instabilities in the clean case. As we are going to show in what follows, the presence of disorder changes this picture significantly.

\section{B. Anderson localized surface states}

If we assume the surface mode is localized as described by (31) one can trace the surface mode completely from the problem. In fact, using (31) and (32) we find that the surface mode is pinned by the edge mode:

$$
\theta(x, t) \approx-\lambda \xi^{2} K u^{-1} \partial_{x}^{2} \phi(x, t)
$$

and therefore, $\rho_{s}(x, t) \propto \partial_{x}^{2} \rho_{e}(x, t)$. The pinning of the surface mode by the edge mode has a rather interesting physical interpretation: in the presence of electronelectron interactions the surface mode is dragged by the edge mode in its motion. The dragging described here has similarities with the Coulomb drag between coupled clean non-chiral Luttinger liquids in quantum wires ${ }^{24,25,26}$ but differs from it in some fundamental ways: (i) the chiral edge state is a persistent current, is not subject to backscattering, and hence can only interact through forward scattering (small momentum transfer), as described in (32); (ii) the non-chiral Luttinger liquid is localized by impurities and hence electrons are not free to move (unless the applied force by the chiral component is greater than a threshold that leads to the depinning of the localized state, a situation not considered here) . Hence, although there is no macroscopic voltage drop along the edge, the force applied by the edge over the surface state will lead to microscopic voltage drops (charge accumulations in the electronic puddles, see Fig. (4). The final picture can be summarized in terms of the scattering of the edge electrons by the potential created by the surface states.

Since the theory described by (20), (31), and (32) is gaussian, the surface states can be exactly traced out of the problem. The effective action for the chiral modes then reads:

$$
S=\frac{m}{4 \pi} \int_{k, \omega} k\left[\omega-v_{\mathrm{E}} k\left(1-g \frac{k^{2}}{k^{2}+\xi^{-2}}\right)\right]|\phi(k, \omega)|^{2}(43)
$$

where,

$$
g=\frac{2 \lambda^{2} K}{m u v_{E}},
$$

is the surface-edge coupling. Notice that the dispersion of the chiral modes is given by:

$$
\omega(k)=v_{\mathrm{E}} k\left(1-g \frac{k^{2}}{k^{2}+\xi^{-2}}\right),
$$

and, hence, for $k \ll \xi^{-1}$ the chiral mode dispersion becomes: $\omega_{k} \approx v_{\mathrm{E}}\left(k-\kappa k^{3}\right)$ where $\kappa=g \xi^{2}$, and for $k \gg \xi^{-1}$ one finds: $\omega_{k} \approx v_{\mathrm{E}}[1-g] k$.

At long wavelengths $\left(k \ll \xi^{-1}\right)$, that is, distances larger than the localization length, the surface mode does not affect the edge mode. The fact that the dispersion at short wavelengths can become negative if $g>1$ indicates the existence of an instability (a quantum critical point) at finite wavevectors. It is easy to see that the dispersion (45) vanishes at $k=k_{c}$ where:

$$
k_{c}^{-1} \sim \xi \sqrt{g-1},
$$

for $g>1$. Therefore, the spectrum of the edge mode becomes negative for $k>k_{c}$ indicating that the edge state becomes unstable. Notice that while disorder (and hence, $\xi$ ) determines the length scale $k_{c}^{-1}$ of the instability, this instability only occurs for a value of $g$ above a critical value $g_{c}=1$ which marks a quantum phase transition in the problem. For $g<g_{c}$ the edge mode is stable but for $g>g_{c}$ and for any amount of disorder there is an instability in the system with characteristic length scale given by (46). The phase diagram of the edge mode as a function of the Luttinger parameter, $K$, and surface-edge coupling, $g$, has, therefore, the structure shown in Fig. 6

In order to numerically estimate the experimental value of this instability, let us consider the case of a weakly interacting surface state $\left(K \approx 1, u \approx v_{\mathrm{S}}\right)$ so that:

$$
g \approx \frac{e^{4}}{t t^{\prime}} \delta
$$

Hence, there is a critical density $\delta_{c}$ such that $g=g_{c}=1$ given by:

$$
\delta_{c} \approx \frac{t t^{\prime}}{e^{4}},
$$

so that $k_{c}^{-1} \rightarrow \infty$ at this point even for small amount of disorder. Clearly, in the absence of disorder $\left(D_{0}=0\right.$, 
see (301) ) $\xi \rightarrow \infty$ we have $k_{c}=0$ at the outset and the instability cannot occur. Notice that $\delta_{c}$ is independent of the disorder, depending only on the ratio between kinetic to Coulomb energies in the system. Although there is uncertainty ${ }^{27}$ on the value of $t^{\prime}$, if we use $t^{\prime} \approx 0.1 t \approx 0.2$ $\mathrm{eV}, e^{2} \approx 16 \mathrm{eV} \AA$, one finds $\delta_{c} \approx 10^{12}-10^{13} \mathrm{~cm}^{-2}$, which is the order of magnitude of carriers in these materials 1.2 .

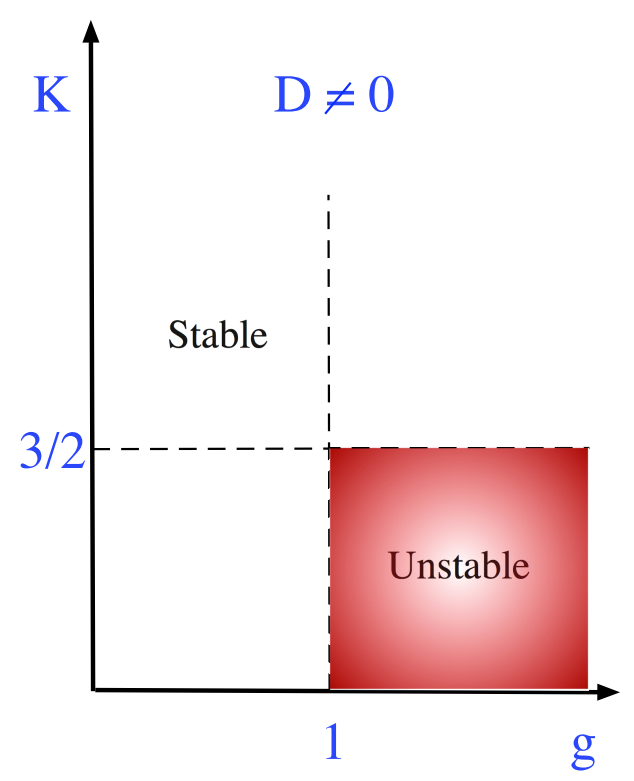

FIG. 6: (color online) Phase diagram of the problem, in the presence of disorder $D_{0} \neq 0$, as a function of the Luttinger liquid parameter, $K$, and surface-edge coupling, $g$.

As shown in Ref. 28], this instability is an indication of a quantum Hall edge reconstruction where new low energy modes are generated at the edge. Edge reconstruction has been proposed to be important for the understanding of IQHE ${ }^{17.29}$ as well as FQHE ${ }^{30}$ in semiconducting devices and for the interpretation of point contact tunneling between a Fermi liquid and a quantum Hall edge. In fact, the current-voltage characteristics for point contact tunneling follows a power law, $I \propto V^{\alpha}$, where the exponent $\alpha$, in the absence of reconstruction, is supposed to be universal and independent of the details of the edge. Nevertheless, recent experiments show a different picture ${ }^{31}$. The discrepancy between theory and experiment can be assigned to edge reconstruction. We expect a similar effect to occur in graphene and graphite.

\section{CONCLUSIONS}

The dragging of the surface mode by the edge mode has also consequences for the magneto-transport. The longitudinal, $\rho_{\mathrm{xx}}$, and Hall, $\rho_{\mathrm{xy}}$, resistivities depend directly on whether the electronic states are localized or not. When the electronic states are localized and the longitudinal conductivity, $\sigma_{\mathrm{xx}}$, vanishes, one has $\rho_{\mathrm{xy}}=1 / \sigma_{\mathrm{QHE}}$ and $\rho_{\mathrm{xx}}=0$ and therefore a perfect quantization of the Hall resistivity in units of $h / e^{2}$.

We have shown that the presence of edge disorder affects directly the physics of the edge states allowing for the possibility of edge reconstruction. The effect is stronger in the FQHE than in the IQHE because of the characteristic energy scales in the problem. In the IQHE the dominant energy scale is the cyclotron energy, $\omega_{c}$. When $\omega_{c}$ is larger than the broadening of the Landau levels due to disorder, the IQHE becomes observable. For a magnetic field $B \approx 6 \mathrm{~T}$ we have $\omega_{c} \approx 1,000 \mathrm{~K}$, which is much larger than the cyclotron energy in conventional semiconducting Hall bars (which is of order of a few $\mathrm{K}$ ). This rather large cyclotron energy (a result of the Dirac dispersion) makes the observation of the IQHE relatively simple $e^{1.2}$.

The situation with the FQHE is very different. For the FQHE what matters is the bulk energy of interaction between the electrons ${ }^{32}$ which is of order of $e^{2} /\left(\epsilon_{0} \ell_{B}\right) \sim$ $\left[e^{2} /\left(\sqrt{2} \epsilon_{0} v_{F}\right)\right] \omega_{c} \sim \omega_{c} / \epsilon_{0}$ where $\epsilon_{0}$ is the dielectric constant of graphene (we have used that $e^{2} /\left(\sqrt{2} v_{F}\right) \sim 1$, see ref. [7]). Because of the presence a back gate in the experiments, we expect screening to be as strong as in ordinary semiconducting devices where $\epsilon_{0} \approx 10$ and hence $e^{2} /\left(\epsilon_{0} \ell_{B}\right) \ll \omega_{c}$. The surface mode localization, and the formation of electronic puddles, lead to changes in the local electrostatic potentials in the Hall bar and affect screening, modifying the bulk electron-electron interactions if the samples are not wide enough (which is the case of the current experiments where the samples are of order of $10 \mu \mathrm{m}$ wide $\mathrm{e}^{1.2}$ ). Therefore, the bulk states of the FQHE will be directly affected by surface state localization leading to a change of the longitudinal conductivity in the system.

In the presence of a surface state, the longitudinal conductivity can be small but finite $\left(\sigma_{\mathrm{xx}} \ll \sigma_{\mathrm{QHE}}\right)$ and strongly dependent on the amount of disorder at the edge. In this case resistivities are given by:

$$
\begin{aligned}
\rho_{\mathrm{xx}} & \approx \rho_{\mathrm{xx}}^{0}\left(\sigma_{\mathrm{xx}} / \sigma_{\mathrm{QHE}}\right)^{2}, \\
\rho_{\mathrm{xy}} & \approx \sigma_{\mathrm{QHE}}^{-1}\left[1-\left(\sigma_{\mathrm{xx}} / \sigma_{\mathrm{QHE}}\right)^{2}\right],
\end{aligned}
$$

where $\rho_{\mathrm{xx}}^{0}=1 / \sigma_{\mathrm{xx}}$. Notice that although there is a large reduction in the longitudinal resistivity $\left(\rho_{\mathrm{xx}} \ll \rho_{\mathrm{xx}}^{0}\right.$ since $\left.\sigma_{\mathrm{xx}} \ll \sigma_{\mathrm{QHE}}\right)$ it is still finite in the "quantum Hall regime". At the same time, the quantization of the Hall resistivity is only partial since it will be spoiled by a factor $\left(\sigma_{\mathrm{xx}} / \sigma_{\mathrm{QHE}}\right)^{2} \ll 1$. This type of effect has been observed in graphite ${ }^{33}$ and we expect it to occur in disordered graphene samples.

In summary, we have studied the integer and fractional quantum Hall effect in graphene taking into account edge and surface modes. We show that although the surface modes are localized by disorder in the absence of a magnetic field, they become delocalized by the edge modes that drag the surface modes in their motion via electron-electron interactions. Our results indicate 
that in this case the Hall edge undergoes a reconstruction leading to a non-universal point contact exponent that depends strongly on the amount of disorder in the system. Furthermore, we also show that a perfect Hall effect is not possible in disordered graphene samples due to the presence of surface modes. Our results show that the Hall resistivity is not quantized and can change significantly from sample to sample depending on disorder and electron-electron interactions.

N. M. R. P. and F. G. acknowledge the Quantum Condensed Matter Visitor's Program at Boston University for support. N. M. R. P. acknowledges Fundação para a Ciência e Tecnologia for a sabbatical grant. A. H. C. N. was supported by the NSF grant DMR-0343790. We thank A. Geim and T. Martin for illuminating discussions.

\section{APPENDIX A: MATRIX ELEMENTS OF $A$}

The matrix $A$ has the following diagonal and offdiagonal elements:

$$
A(1,1)=q v_{S} \cos ^{2} \phi \cosh ^{2} \theta-2 V(q) \cos \phi(\sin \phi+\cos \phi \sinh \theta) \cosh \theta+E(q)\left(\sin ^{2} \phi+\cos ^{2} \phi \sinh ^{2} \theta\right)
$$

$$
\begin{aligned}
A(2,2) & =\left(q v_{S} \cosh ^{2} \theta \sin ^{2} \phi+\tilde{V}(q) \cosh \theta\left(\sin (2 \phi)-2 \sin ^{2} \phi \sinh \theta\right)+E(q)\left(\cos ^{2} \phi+\sin ^{2} \phi \sinh ^{2} \theta\right)\right) \cosh ^{2} \eta \\
& +\sinh (2 \eta)\left(\left(\tilde{V}(q) \cos \phi+\left(q v_{S}+E(q)\right) \cosh \theta \sin \phi\right) \sinh \theta-\tilde{V}(q) \cosh (2 \theta) \sin \phi\right) \\
& +\sinh ^{2} \eta\left(E(q) \cosh ^{2} \theta-2 \tilde{V}(q) \sinh \theta \cosh \theta+q v_{S} \sinh ^{2} \theta\right)
\end{aligned}
$$

$$
A(3,3)=q v_{S} \cos ^{2} \phi \cosh ^{2} \theta-2 \tilde{V}(q) \cos \phi(\sin \phi+\cos \phi \sinh \theta) \cosh \theta+E(q)\left(\sin ^{2} \phi+\cos ^{2} \phi \sinh ^{2} \theta\right)
$$

$$
\begin{aligned}
A(1,2) & =\tilde{V}(q) \cos (2 \phi) \cosh \eta \cosh \theta+\sinh \eta\left(\left(\left(q v_{S}+E(q)\right) \cos \phi \cosh \theta-\tilde{V}(q) \sin \phi\right) \sinh \theta-\tilde{V}(q) \cos \phi \cosh (2 \theta)\right) \\
& +\frac{1}{4} \cosh \eta \sin (2 \phi)\left(q v_{S}-3 E(q)+\left(q \tilde{v}_{S}+E(q)\right) \cosh (2 \theta)-2 \tilde{V}(q) \sinh (2 \theta)\right)
\end{aligned}
$$

$$
\begin{aligned}
A(1,3) & =-\tilde{V}(q) \cos (2 \phi) \cosh \theta \sinh \eta-\frac{1}{4} \sin (2 \phi)\left(q v_{S}-3 E(q)+\left(q v_{S}+E(q)\right) \cosh (2 \theta)\right. \\
& -2 \tilde{V}(q) \sinh (2 \theta)) \sinh \eta+\cosh \eta\left(\tilde{V}(q)(\cos \phi \cosh (2 \theta)+\sin \phi \sinh \theta)-\left(q v_{S}+E(q)\right) \cos \phi \cosh \theta \sinh \theta\right)
\end{aligned}
$$

$$
\begin{aligned}
A(2,3) & =\frac{1}{8} \sinh (2 \eta)\left(2\left(q v_{S}-3 E(q)\right) \cos ^{2} \phi+\left(q v_{S}+E(q)\right)(\cos (2 \phi)-3) \cosh (2 \theta)-4 \tilde{V}(q) \cosh \theta \sin (2 \phi)\right. \\
& -2 \tilde{V}(q)(\cos (2 \phi)-3) \sinh (2 \theta))+\frac{1}{2} \cosh (2 \eta)(2 \tilde{V}(q) \cosh (2 \theta) \sin \phi \\
& \left.-\left(q v_{S}+E(q)\right) \sinh (2 \theta) \sin \phi-2 \tilde{V}(q) \cos \phi \sinh \theta\right)
\end{aligned}
$$

${ }^{1}$ K. S. Novoselov et al., Nature 438, 197 (2005).

${ }^{2}$ Y. Zhang et al., Nature 438, 201 (2005).

3 D. P. DiVincenzo and E. J. Mele, Phys.Rev.B 29, 1685 (1984).

4 J. González, F. Guinea, and M. A. H. Vozmediano, Phys.
Rev. Lett. 77, 3589 (1996).

5 See, for instance, P. J. Hirschfeld, and W. A. Atkinson, J. Low. Temp. Phys. 126, 881 (2002).

6 See papers in Carbon-Based Magnetism: an overview of metal free carbon-based compounds and materials, T. 
Makarova and F. Palacio, eds. (Elsevier, Amsterdam, 2005).

7 N.M.R. Peres, F. Guinea, and A. H. Castro Neto, Phys. Rev. B 72, 174406 (2005).

8 N.M.R. Peres, F. Guinea, and A. H. Castro Neto, cond-mat/0506709 and cond-mat/0512091

9 For $B=12 \mathrm{~T}$ we find $\hbar \omega_{c} \approx 0.142 \mathrm{eV}(\approx 1651 \mathrm{~K})$ and $g \mu_{B} B \approx 7 \times 10^{-4} \mathrm{eV}(\approx 8 \mathrm{~K})$.

10 B. I. Halperin, Phys. Rev. B 25, 2185 (1982).

11 V. P. Gusynin, and S. G. Sharapov, Phys. Rev. Lett. 95, 146801 (2005).

12 K. Wakayabashi, and M. Sigrist, Phys.Rev.Lett. 84, 3390 (2000); K. Wakayabashi, Phys. Rev.B 64, 125428 (2001).

13 Notice that strictly speaking there is no surface in a finite graphene layer but a $1 \mathrm{D}$ edge. In order to make distinction between QHE edge states generated by a magnetic field and graphene edge states we call the latter surface states.

14 N. M. R. Peres, A. H. Castro Neto, and F. Guinea, cond-mat/0512476

15 X.-G. Wen, Int. J. Mod. Phys. B 6, 1711 (1992).

16 J. Voit, Rep. Prog. Phys. 58, 977 (1995).

17 C. C. Chamon, and X.-G. Wen, Phys. Rev. B 49, 8227 (1994).

18 Y. Kobayashi, K.-I. Fukui, T. Enoki, K. Kusakabe, and Y. Kaburagi, cond-mat/0503472.

19 T. Giamarchi, and H. J. Schulz, Phys. Rev. B 37, 325 (1988).

20 Gerald D. Mahan, Many-Particle Physics, 2nd. Edition, (Plenum, New York, 1990).
21 O. Heinonen, and Sebastian Eggert, Phys. Rev. Lett. 77, 358 (1996).

22 J. H. P. Colpa, Physica A 93, 327 (1978).

23 The matrix element $T_{33}$ of the transformation $T$ given in Ref. 21] should read $\sin \phi \sinh \theta \sinh \eta+\cosh \theta \cosh \eta$.

${ }^{24}$ Yu. V. Nazarov, and D. V. Averin, Phys. Rev. Lett. 81, 653 (1998).

25 R. Klesse, and A. Stern, Phys. Rev. B 62, 16912 (2000).

26 M. Pustilnik, E. G. Mishchenko, L. I. Glazman, and A. V. Andreev, Phys. Rev. Lett. 91, 126805 (2003).

27 Band-structure parametrization usually assumes $t^{\prime}=0$ (see, for instance, J.-C. Charlier et al., Phys. Rev. B 43, 4579 (1991)).

${ }^{28}$ K. Yang, Phys. Rev. Lett. 91, 036802 (2003).

29 A. H. MacDonald et al., Aust. J. Phys. 46, 345 (1993).

30 X. Wan, K. Yang, and E. H. Rezayi, Phys. Rev. Lett. 88, 056802 (2002).

31 A. M. Chang, L. N. Pfeiffer, and K. W. West, Phys. Rev. Lett. 77, 2538 (1996); M. Grayson et al., Phys. Rev. Lett. 80, 1062 (1998); A. M. Chang et al., Phys. Rev. Lett. 86, 14 (2001); M. Hilke et al., Phys. Rev. Lett. 87, 186806 (2001).

32 G. Murthy, and R. Shankar, Rev. Mod. Phys. 75, 1101 (2003).

33 S. Uji, J. S. Brooks, and Y. Iye, Physica B 246-247, 299 (1998); Y. Kopelevich et al., Phys. Rev. Lett. 90, 156402 (2003). 